\title{
Real-space density profile reconstruction of stacked voids
}

\author{
Alice Pisani ${ }^{1,2}$, P. Sutter ${ }^{1,2,3}$, G. Lavaux ${ }^{1,2}$ and B. Wandelt ${ }^{1,2,4,5}$ \\ ${ }^{1}$ Sorbonne Universités, UPMC Univ Paris 06, UMR7095, F-75014, Paris, France \\ ${ }^{2}$ CNRS, UMR7095, Institut d'Astrophysique de Paris, F-75014, Paris, France \\ ${ }^{3}$ Center for Cosmology and AstroParticle Physics, Ohio State University, Columbus, USA \\ ${ }^{4}$ Department of Physics, University of Illinois at Urbana-Champaign, Urbana, USA \\ ${ }^{5}$ Department of Astronomy, University of Illinois at Urbana-Champaign, Urbana, USA
}

\begin{abstract}
Modern surveys allow us to access to high quality large scale structure measurements. In this framework, cosmic voids appear as a new potential probe of Cosmology. We discuss the use of cosmic voids as standard spheres and their capacity to constrain new physics, dark energy and cosmological models. We introduce the Alcock-Paczyński test and its use with voids. We discuss the main difficulties in treating with cosmic voids: redshift-space distortions, the sparsity of data, and peculiar velocities. We present a method to reconstruct the spherical density profiles of void stacks in real space, without redshift-space distortions. We show its application to a toy model and a dark matter simulation; as well as a first application to reconstruct real cosmic void stacks density profiles in real space from the Sloan Digital Sky Survey.
\end{abstract}

Keywords. cosmology: large-scale structure of universe, methods: dark energy, Abel inverse

\section{Introduction}

The large scale structure of the Universe is an incredibly powerful tool to constrain cosmic evolution. At large scales, clusters of galaxies, voids and filaments shape the Universe - it is the Cosmic Web. Cosmic voids, discovered in 1978 (Gregory \& Thompson, 1978; Jõeveer et al., 1978), are under-dense regions in the Universe with sizes from ten to hundreds of Mpcs. Until very recently, due to the difficulty of extracting data from low density zones, the potential of voids has been under-explored.

With the increasing precision and quality of modern surveys we now have access to high quality large scale structure measurements: the appeal of cosmic voids becomes thus considerable. Being devoid of matter, cosmic voids might be mainly composed of dark energy (Bos et al., 2012) — which strongly justifies their importance for Cosmology, as dark energy is believed to be $\sim 70 \%$ of the Universe and we are still not able to understand it. The efforts of cosmologists seem to converge to a model (called $\Lambda C D M$ ) that leaves many unknowns. The nature - and we could say even more, the existence — of dark energy remains a mystery; and so does the nature of dark matter. In this framework cosmic voids appear as a new tool to use in our research of a correct cosmological model.

Cosmic voids have a much simpler evolution compared to high density zones of the Universe and, as such, constitute a promising laboratory to test dark energy, constrain cosmic expansion and discriminate between cosmological models such as modified gravity models (e. g. Spolyar et al., 2013). The difficulties in extracting information from voids are: the irregular shape of voids, the sparsity of galaxies and the presence of redshift-space distortions (RSD).

In the first part we discuss the use of voids for cosmology, the second part presents the key idea to overcome RSD and its test on a toy model. In the third part we illustrate the 
application of the algorithm to a simulation and we present the first ever real-space density profiles of stacked voids from SDSS DR7 data, reconstructed without any assumption about RSD. Finally we conclude discussing future applications of the algorithm.

\section{Cosmic voids for Cosmology}

The shape of cosmic voids is not regular. Cosmic voids are then difficult objects to study because of their irregular shape and of the sparsity of data: galaxies are mostly concentrated in filaments or clusters of galaxies. To overcome both the irregularity of the shape and the small amount of data, Lavaux and Wandelt (2012) suggested a new approach consisting in the stacking of voids. In a homogeneous and isotropic Universe, the average real-space shape of cosmic voids - obtained by stacking - is spherical. Because of its spherical shape, the stacked void can be used to extract cosmological information. To reach such goal, we use void catalogues to obtain stacked voids at different redshift and of different sizes. Then, the spherical profiles of stacked voids can be used as standard spheres, necessary tools for the so-called Alcock-Paczyński test.

The Alcock-Paczyński test (Alcock and Paczyński, 1979), proposed in 1978 by Charles Alcock and Bohdan Paczyński, compares the shape of a standard sphere in real and redshift space to constrain the expansion of the Universe. When applied to voids (Sutter et al., 2014d), the test compares the shape of the stacked distorted void in redshift space and of the stacked spherical void in real space to obtain information about the expansion of the universe. It uses the void as a standard sphere.

The term standard sphere is an analogy with the idea of standard candle or standard ruler. A standard candle (ruler) is an object of known luminosity (length) used to measure the expansion of the universe through the difference between the observed and the expected luminosity (length). For a standard sphere the known quantity is the diameter of the sphere, that we measure along and perpendicularly to the line of sight direction. Unfortunately, although void stacks would be perfect as standard spheres, we cannot perform a precise Alcock-Paczyński test without the real space profile of voids (Pisani et al. (2014a)): observations will always give us the shape in redshift space.

Indeed, surveys measure the position of galaxies in redshift space. Since our universe is expanding, all galaxies are redshifted due to the expansion of space. To this is added the redshift caused by the peculiar motion of the galaxy. Only the line of sight component of velocity affects the galaxy redshift (Hamilton, 1998). In the framework of voids, it means that the real-space spherical shape of voids is distorted in redshift space: we do not measure a spherical shape of the stacked void, we measure a distorted void in redshift space. In the next part we describe the key idea to obtain the shape of voids in real space.

\section{Overcoming RSD: real-space density reconstruction}

To extract cosmological information from real stacked voids, we need their shape in real space. It is fundamental to recover the real-space density without any assumption about RSD, otherwise the cosmological results would not be independent from the model used for RSD.

The key idea is that RSD only affect the line of sight component of the galaxy positions. If we were able to reconstruct the shape of the stacked void without using this component - that is from the projection on the $\mathrm{x}-\mathrm{y}$ plane - we could obtain the void in real space (see figure 1, left).

We consider the redshift-space density of the void (number of galaxies per volume element), we then project it by counting all the galaxies in bins in the $\mathrm{x}-\mathrm{y}$ plane and 

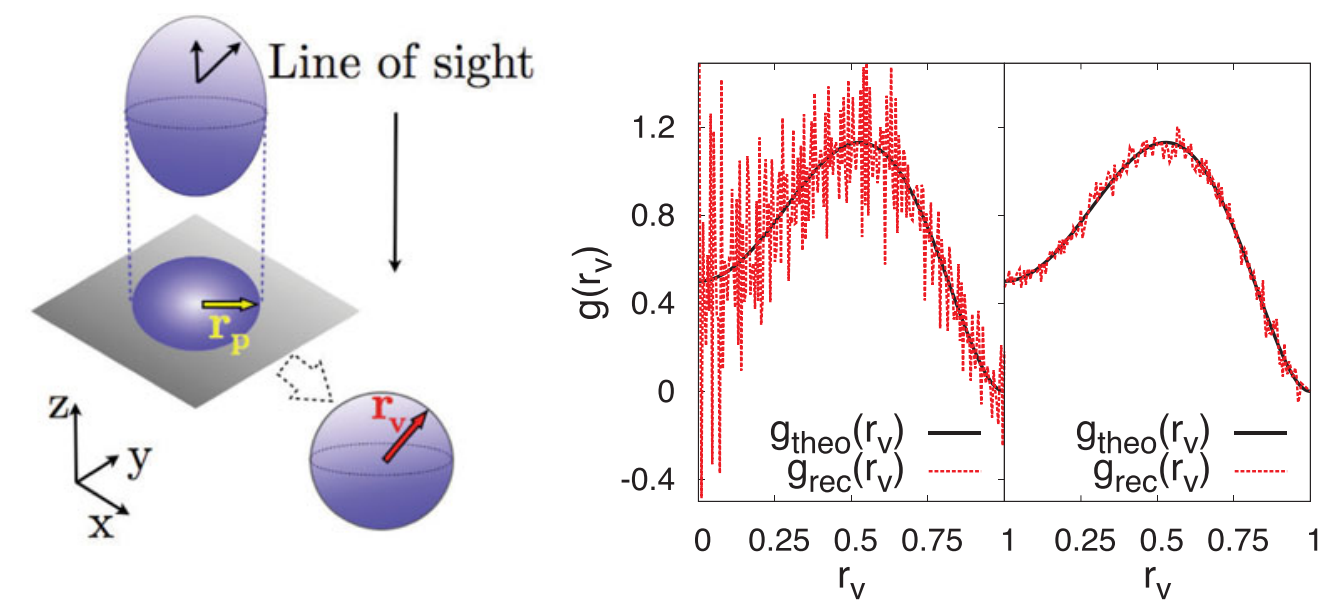

Figure 1. Left: The key idea to obtain the density profiles of voids in real space. Right: Reconstruction of the toy model density profile, without (first plot) and with (second plot) the regularisation algorithm for the inversion. Reproduced from Pisani et al. (2014a)

obtain the projected density. To obtain the spherical density profile of the void from the projection, we use Abel inverse transform (Abel, 1842):

$$
g\left(r_{\mathrm{v}}\right)=-\frac{1}{\pi} \int_{r_{\mathrm{v}}}^{1} \frac{I^{\prime}\left(r_{\mathrm{p}}\right)}{\sqrt{r_{\mathrm{p}}^{2}-r_{\mathrm{v}}^{2}}} d r_{\mathrm{p}}
$$

where $g\left(r_{\mathrm{v}}\right)$ is the spherical density profile of galaxies in real space we aim to reconstruct, $r_{\mathrm{v}}=\sqrt{x^{2}+y^{2}+z^{2}}$ is the void's radius, $r_{\mathrm{p}}=\sqrt{x^{2}+y^{2}}$ is the radius of the projection onto a plane perpendicular to the line of sight and $I\left(r_{\mathrm{p}}\right)$ is the projected density.

The problem is that the Abel inverse transform, although well mathematically defined (see equation 3.1), is strongly ill-conditioned: if there is some noise in the input function $I\left(r_{\mathrm{p}}\right)$ (of which $I^{\prime}\left(r_{\mathrm{p}}\right)$ is the derivative with respect to $r_{\mathrm{p}}$ ), the reconstruction will be dominated by noise. To overcome the problem of ill-conditioning we have implemented for the case of voids a polynomial regularization of the inversion (Li et al., 2007), allowing to obtain the spherical density profiles of voids in real space.

We test the reconstruction with a toy model: we artificially distort a void stack with a simple model of RSD and we reconstruct the profile in real space. As a pedagogical illustration, we show the reconstruction of the density without any regularization algorithm for the Abel inverse transform and we compare it with the reconstruction that uses our algorithm (see figure 1, right; note that the void is a toy model and the density profile does not reflect the real shape of a void). The ill-conditioning of the reconstruction is well controlled. The next section presents the results with a dark matter particle simulation and real data from SDSS.

\section{Results: simulation and SDSS data}

In this part, we show the result of the reconstruction (figure 2) of a stacked void from a dark matter simulation. More details can be found in Pisani et al. (2014a). As a first application of the algorithm to real data, we reconstruct the density profiles of stacked voids from the void catalogue presented in Sutter et al. (2012). We show in figure 2 a reconstructed profile for a real stacked void. Despite the noise present in real data, 

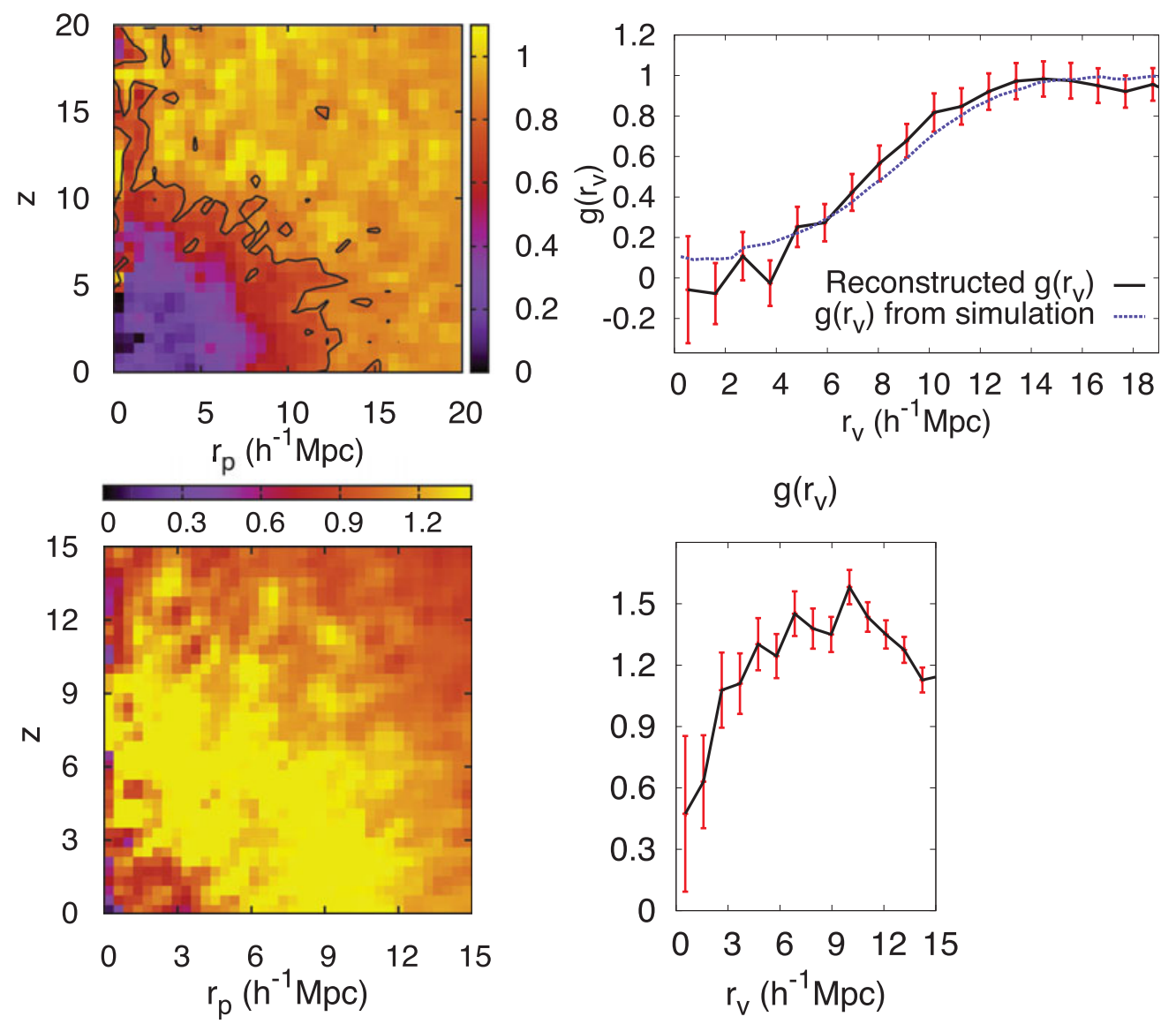

Figure 2. From left to right: density in redshift space from the simulation; comparison of the reconstructed density profile with the profile in real space from the simulation; density in redshift space for a 5-15 $h^{-1} M p c$ stacked void from SDSS and reconstructed density profile in real space. Reproduced from Pisani et al. (2014a).

the algorithm is capable to obtain the density profile of the void, overcoming the illconditioning of the inverse. To conclude, in the next section we briefly discuss future applications for the reconstruction of the density profiles.

\section{Conclusion: modelling systematics for the future}

We developed an algorithm to reconstruct density profiles of voids in real space without any assumption about RSD. The knowledge of the real space shape of the void is instrumental to improve the application of the Alcock-Paczyński test, since the method for the test is based on the real-space profile of void stacks. Additionally to the real-space modelling, information about peculiar velocity effects on cosmic voids would also enhance the understanding of systematics effects in their use for Cosmology and in the study of void evolution. An analysis of the effects of velocities promises to improve the precision of cosmological constraints from voids. The comparison of mock catalogues of galaxies with and without peculiar velocities allows to obtain guidelines to master such effects (see Pisani et al. (2014b)). 
The density profiles of stacked voids in real space open the way to better constrain the value of the Hubble constant, cosmological models and new physics on current and future data sets.

\section{Acknowledgements}

AP would like to express her gratitude to the organizers for the opportunity to deliver this talk. The authors acknowledge support from BDW's Chaire Internationale at UPMC and ANR-10-CEXC-004- 01).

\section{References}

Gregory S. A. \& Thompson L. A., 1978, ApJ, 222, 784

Jõeveer, M., Einasto, J., \& Tago, E., MNRAS, 185:357-370, November 1978.

Bos, E., van de Weygaert, R., Dolag, K., \& Pettorino, V., MNRAS, 426(1)440-461, 2012.

Spolyar, D., Sahlén, M., \& Silk, J., Physical Review Letters, 111(24):241103, Dec., 2013.

Lavaux, G. \& Wandelt, B. D. ApJ, 754:109, Aug. 2012.

Alcock, C. \& Paczyński, B. Nature, 281:358, Oct.1979.

Sutter, P., Pisani, A., \& Wandelt, B. D. MNRAS, Vol. 443, Issue 4, p. 2983-2990, 2014d

Hamilton, A. J. S. vol. 231 of Astroph. and Space Science Library, p.185, 1998

Abel, N. H. Oeuvres Completes, 1842, Ed. L. Sylow \& S. Lie, Johnson Reprint Corp. 1988

Li, X.-F., Huang, L., \& Huang, Y. Journal of Physics A Math. Gen., 40:347-360, Jan. 2007

Pisani, A., Lavaux, G., Sutter, P. M., \& Wandelt, B. D. MNRAS, 443:3238-3250, Oct. 2014a

Sutter, P. M., Lavaux, G., Wandelt, B. D., \& Weinberg, D. H. ApJ, 761:44, Dec. 2012.

Pisani, A., Sutter, P. M., \& Wandelt, B. D. in prep., 2014b. 